SOUTH EAST EUROPE

Transnational Cooperation Programme \section{SNAPDS SEE}

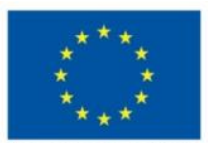

Programme co-funded by the EUROPEAN UNION

Daten und Analyse-Methoden für Baurohstoff-Planung: Zur Unterstützung von Best Practices für eine nachhaltige Baurohstoff - Planung

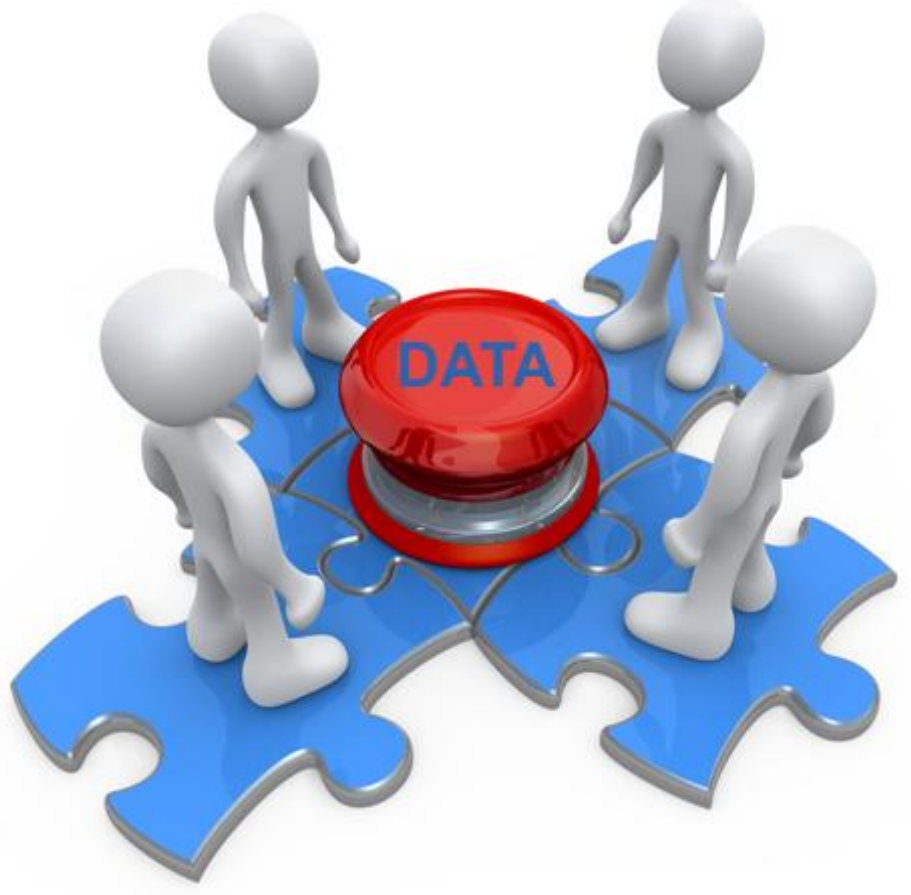

May 2014

Technical University of Crete
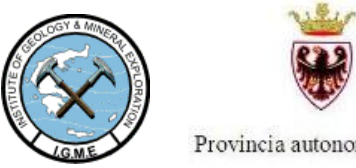

Provincia autonoma di Trento
POLITECNICO DI TORINO 
Bezogen auf:

die Berichte des Arbeitsparkets 4 des SNAP-SEE-Projekt "Sustainable Aggregates Planning in South East Europe" (SEE/D/0167/2.4/X)

Resultat 4.3: Handbuch zur Datenanalyse und Methoden für die BaurohstoffPlanung: Ein gemeinsames Handbuch für Planungsbehörden in der SEE-Region

Website:http://www.snapsee.eu

\section{Publication Information}

Editing\& Layout:

Cover Design:

Printing:

Year of Edition
Foteini Stathogianni

Stelios Mavrigiannakis

Geological Survey of Slovenia

2014

\section{Disclaimer}

Dieses Snap-SEE Veröffentlichung spiegelt nur die Meinung der Autoren. Die Verwaltungsbehörde des Programms Süd-Ost-Europa grenzüberschreitende Zusammenarbeit ist nicht verantwortlich für jegliche Verwendung der darin enthaltenen Informationen. (C) SNAP-SEE-Projekt 2014 


\begin{tabular}{|c|c|}
\hline & $\begin{array}{l}\text { Agioutantis, Zach,Dr.,Technical University of Crete (TUC), Greece } \\
\text { Komnitsas, Kostas, Dr.,Technical University of Crete (TUC), Greece } \\
\text { Steiakakis, Manolis, Dr., Technical University of Crete (TUC), Greece } \\
\text { Mavrigiannakis, Stelios, Technical University of Crete (TUC), Greece } \\
\text { Stathogianni, Foteini, Technical University of Crete (TUC), Greece }\end{array}$ \\
\hline & $\begin{array}{l}\text { Chalkiopoulou, Fotini, Institute of Geology \& Mineral Exploration (IGME), } \\
\text { Greece } \\
\text { Hatzilazaridou, Kiki, Institute of Geology \& Mineral Exploration (IGME), } \\
\text { Greece }\end{array}$ \\
\hline Provincia autonoma di Trento & $\begin{array}{l}\text { Moltrer, Alessandro, Autonomous province of Trento- Environmental } \\
\text { Protection Provincial Agency, Italy } \\
\text { Valbusa, Michele, Autonomous province of Trento- Environmental Pro- } \\
\text { tection Provincial Agency, Italy }\end{array}$ \\
\hline & $\begin{array}{l}\text { Bobba, Silvia, Politecnico di Torino, Italy } \\
\text { Blengini, Gian Andrea, Dr., Politecnico di Torino, Italy }\end{array}$ \\
\hline
\end{tabular}

Reviewers

\begin{tabular}{ll}
\hline Name & Affiliation \\
\hline $\begin{array}{l}\text { Shields, Deborah J., Dr. } \\
\text { O'Brien, Jim }\end{array}$ & $\begin{array}{l}\text { Montanuniversität Leoben (University of Leoben), Austria } \\
\text { Former President of the European Aggregates Association, Ireland } \\
\text { Simic, Vladimir, Dr. }\end{array}$ \\
& $\begin{array}{l}\text { University of Belgrade, Faculty of Mining and Geology, Serbia } \\
\text { Acknowledgment }\end{array}$ \\
The SNAP-SEE project partners would like to thank the European Commission for funding this project which \\
led to a fruitful cooperation between partners sharing similar issues and a common vision, and allowed the \\
development of this handbook.
\end{tabular}

\section{Digital Object Identifier (DOI)}

doi: 10.5474/snapsee-WP4-AT

\section{Citation}

This handbook should be cited in the bibliography as follows: Agioutantis Z. et al. (2014), Handbook on Data and Analysis Methodologies for Aggregates Planning: A joint manual for planning authorities in SEE region, SNAPSEE, 2014, doi: 10.5474/snapsee-WP4-AT 


\section{Vorwort}

1

Einleitung

1.1 Das Bedürfnis einer Baurohstoff-Planung 8

1.2 Rahmen und Struktur des Handbuches 9

$\begin{array}{lll}1.3 & \text { Baurohstoff-Kategorien } & 10\end{array}$

$2 \quad$ Bedeutung der Daten und Analyseverfahren für Planung 12

$2.1 \quad$ Generelle Berücksichtigung 13

2.2 Bedeutung der Daten für Nachhaltiges Planen 13

2.3 Datenverfügbarkeit in den SEE-Ländern / Regionen 14

$3 \quad$ Organisieren der Informationen 17

3.1 Planungskomponenten 18

3.2 Einrichten der Informationsmatrix 18

$\begin{array}{lll}3.3 & \text { Begrenzungen } & 21\end{array}$

$4 \quad$ Ermittlung des Datenbedarfs $\quad 22$

4.1 Datenformat 23

4.2 Identifizierung der wichtigsten Datensätze für Primärbaurohstoffe 23

4.2.1 Daten für die Sicherung mineralischer Baurohstoffe in Raumnutzungs- 24 plänen

4.2.2. Daten um die Wissenbasis der geologischen Verteilung geeigneter 25 primärer Ressourcen zu verbessern

4.2.3. Daten zu Produktion/produktionskapazität, Verbrauch und Im- 26 port/Export

4.2.4. Informationen 26

4.2.5. Daten, um die Auswirkungen des Transports von Zuschlagstoffen aus $\quad 27$ der Abbaustätte zu den Verbrauchermärkten zu bewerten

4.2.6. Daten über die Einhaltung der gesamtwirtschaflichen Ressourcen mit 27 technischen Spezifikationen

4.2.7 Daten für die Nachfrageprognosen 28

4.3 Identifizierung von Hauptdatensätzen für Sekundäre Baurohstoffe 29

$5 \quad$ Analysemethoden 33

$5.1 \quad$ Einleitung 34

$\begin{array}{lll}5.2 & \text { Datenvalidierung } & 34\end{array}$ 
5.3 Materialflussrechnung und Analysis 36

$5.4 \quad$ Nachfrageprognose 36

5.4.1 Kausalnachfrageprognosen auf Grundlage lokaler Landnutzungspla- 38 nung

5.4.2. Kausalbedarfsprognosen auf Basis von Bevölkerungszahl oder Wirtschaftstätigkeit

5.4.3. Zeitreihen Prognosen basierend auf Produktionsstatistiken 37

5.4.4. Zeitreihen Prognosen basierend auf Verbrauchsstatistik 38

$6 \quad$ Empfehlungen $\quad 39$

6.1 Generelle Empfehlungen 40

6.2 Empfehlungen für Daten und Datenanalysemethoden 40

$7 \quad$ Literaturliste

42 


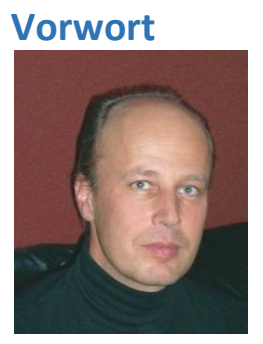

Die Länder in Südosteuropa (SEE) sind reich an Baurohstoffe, also Schotter, Kies, Sand und anderen Rohstoffen die in der Bauindustrie verwendet werden. Allerdings sind diese Mittel sind nicht gleichmäßig verteilt. Weiterhin wird der Zugriff auf diese Ressourcen immer schwieriger aufgrund Infrastrukturentwicklung und Umweltbelastungen. Als Baurohstoffe sind sie zu massiv, ist der Transport zu teuer, daher ist der Zugang zu lokalen Ressourcen entscheidend. In der SEE-Region wird wahrscheinlich die Nachfrage von Baurohstoffen bis 2020 um 50\% ansteigen, um den wachsenden Infrastrukturbedarf umfassend Rechnung zu tragen.

Daher ist ein nachhaltiges Gesamtressourcenmanagement (SARM) und ein nachhaltiges Versorgung Mix (SSM) von Baurohstoffen erforderlich, um die Ressourceneffizienz zu verbessern und die nachhaltige Entwicklung. Die Umsetzung der SARM- und SSM-Konzepte erfordert eine Unterstützung der Rohstoffplanungspolitik. Aufgrund der regionalen Unterschiede und historische Entwicklung, unterscheiden sich die Ansätze der Baurohstoffpolitik, der Planung und des Management innerhalb und zwischen den SEE-Länder. Herausforderungen beziehen sich auf Maßnahmen und Pläne, welche die Baurohstoffe Verwaltung beeinflussen, die unter vielen verschiedenen Rechtsdokumenten verteilt sind, so dass die Koordination und ein umfassendes Verständnis schwierig ist. Es gibt auch einen Mangel der Koordination bei der Planung für die Versorgung aus primären und sekundären Baurohstoffe. Viele SEELänder haben nicht genügend Daten für die Baurohstoffe-Planung, eine unzureichende Kapazität und Kompetenz für die Adressierung entweder primären und / oder sekundären Baurohstoffe Planung, und eine unzureichende Beteiligung der Betroffenen an der Entwicklung der Baurohstoffe-Pläne. Das SNAP-SEE-Projekt hat diese Defizite angesprochen, zur Förderung eines Wachstums einer lebendigen, verantwortlichen und nachhaltigen Baurohstoff -Industrie in der Region.

Der spezifische Zweck von SNAP in Südosteuropa (SEE-SNAP) war die Erstellung und Verbreitung einer Toolbox für Baurohstoff-Planung für Regierungen und Interessengruppen, um ihre Baurohstoff-Planung und Managementprozesse zu verbessern. Das SNAP-SEE Projekt baut auf den Ergebnissen des Projekts "Nachhaltige Baurohstoffe Resource Management" (Sarma) auf, ein vorhergehendes Projekt, gefördertert vom Transnational Cooperation (http://www.sarma.eu). SNAP-SEE wurde ebenfalls von der EU in Südosteuropa finanziert (SEE) Programm Transnationale Zusammenarbeit (SNAP-SEE, SEE / D / 0167 / 2.4 / X) und hatte 27 Partner aus 12 SEE-Ländern und der Türkei. Die Montanuniversitaet Leoben, Österreich, war der federführende Partner. SNAP-SEE war ein 2-Jahres-Projekt, das im November 2014 endete. 
Die SNAP-SEE-Toolbox für Baurohstoffe Planning umfasst 4 Produkte, die miteinander verbunden sind und sich gegenseitig stützen.

1. Eine Vision von Best Practices für Baurohstoff-Planung in Südosteuropa (Vision of Best Practices for Aggregates Planning in South East Europe) "Best Practices" bedeuten eine Vision für einen Übergang zur integrierten, umfassenden nachhaltigen Planung in den SEE-Ländern. Es enthält Diskussionen über die Themen, die angesprochen werden, die in Richtung nachhaltige Planung führen, sowie zur Überprüfung der Komponenten eines nachhaltigen Plan.

\section{Wie man nachhaltigen Baurohstoff-Plan erstellt}

Das 'Wie' Dokument stellt einen Fahrplan für die Planung, einschließlich Diskussionen über den Planungsprozess selbst und den verschiedenen Schritten. Beispiele für Planungsmodule sind vorgesehen, die die Prinzipien, Ansätze und notwendige Aktionen verkörpern, um die Ziele der Visionen zu erreichen.

3. Konsultation der Betroffenen, bei Anwendung der besten Praktiken der Nachhaltigen Baurohstoff-Planung (Consulting Stakeholders when Applying Best Practices in Sustainable Aggregates Planning)

Das "Konsultation" Dokument bietet eine Schritt-für-Schritt-Anleitung für die Planung und Durchführung von Stakeholder-Konsultationen, um zu gewährleisten, dass die Industrie, Behörden, Nicht-Regierungs-Organisationen und der Zivilgesellschaft einen Input bringen.

\section{Daten und Analysen zur Unterstützung von Best Practices in nachhaltigen} Baurohstoffe Planung (Data and Analysis in Support of Best Practices in Sustainable Aggregates Planning)

Dieses Dokument beschreibt die verschiedenen Arten von Daten, die wesentlichen Grundinformationen für den Planungsprozess. Methoden zur Validierung und Daten-Analyse werden bereitgestellt, darunter Ansätze zur Vorhersage der Nachfrage.

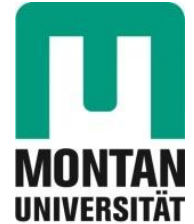

WWW.UNILEOBEN.AC.AT
Project Koordinator

Guenter Tiess

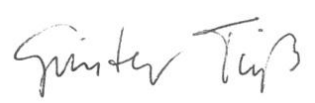

Montanuniversitaet Leoben

(University of Leoben) 


\section{Einleitung}

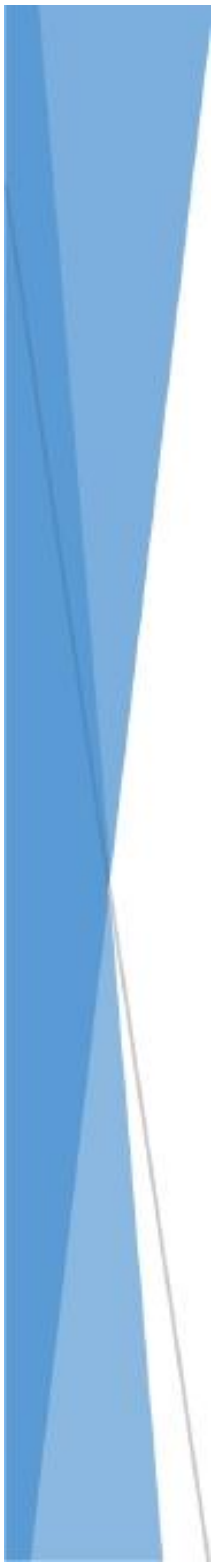

Die Sicherstellung einer nachhaltigen Baurohstoffe Versorgung ist eine wichtige Herausforderung aufgrund der begrenzten Verfügbarkeit der natürlichen Ressourcen. Aufgrund der regionalen Unterschiede und historischen Entwicklung gibt es unterschiedliche Ansätze zur Baurohstoff-Politik, Planung und Ressourcenmanagement in Süd-Ost-Europa. Dies unterminiert die Ressourceneffizienz und wirtschaftlichn Entwicklung in der Region. Erfolgreiche Planung für das Baurohstoff-Management und die Versorgung von primären und sekundären Baurohstoffen erfordert eine umfassende Informationen.

Ohne ausreichende Informationen Ansonsten kann es schwierig oder unmöglich sein, die Ziele des Plans, unrealistisch oder sogar kontraproduktiv, vor allem in Bezug auf Ressourceneffizienz.

Es ist auch der Fall, dass in den meisten südosteuropäischen Ländern, sekundäre Baurohstoffe in der Planung für Baurohstoff-Versorgung nicht berücksichtigt werden, zB Volumina von Baureststoffen. Um effektive und nachhaltige nationale / regionale Pläne für primäre und sekundäre Baurohstoffe zu realisieren, muss die gesamte Palette der benötigten Daten identifiziert werden. 


\subsection{Das Bedürfnis einer Baurohstoff-Planung}

Baurohstoffe (Schotter, Kies und Sand) sind essentiell für Wohn-, soziale und kommerzielle Infrastruktur einer modernen Europäischen Gesellschaft. Europa verbraucht derzeit rund 3 Milliarden Tonnen Baurohstoffe pro Jahr; hat rund 26000 Abbaue. Der Baurohstoffebedarf in Europa reicht von 2 bis 16 Tonnen pro Kopf (UEPG, 2013). Tabelle 1 zeigt den geschätzten Verbrauch für SEE Länder, die Durchschnittswerte betragen 3,5 Tonnen pro Kopf.

Tabelle 1.1: Geschätzte SEE Region Tonnagen (UEPG, 2013)

\begin{tabular}{|l|c|c|c|}
\hline & $\begin{array}{c}\text { Consumption } \\
\text { (million tonnes) }\end{array}$ & $\begin{array}{c}\text { population } \\
\text { (million people) }\end{array}$ & Tonnes/capita \\
\hline Albania & 10 & 3.2 & 3.1 \\
\hline $\begin{array}{l}\text { Bosnia - Her- } \\
\text { zegovina }\end{array}$ & 12 & 3.8 & 3.2 \\
\hline Bulgaria & 29 & 7.6 & 3.8 \\
\hline Croatia & 13 & 4.4 & 3.0 \\
\hline Greece & 25 & 11.3 & 2.2 \\
\hline Hungary & 36 & 10.0 & 3.6 \\
\hline FYROM & 6 & 2.0 & 3.0 \\
\hline Montenegro & 2 & 0.6 & 3.3 \\
\hline Romania & 96 & 22.0 & 4.4 \\
\hline Serbia & 17 & 7.3 & 2.3 \\
\hline Slovakia & 23 & 5.4 & 4.3 \\
\hline Slovenia & 9 & 2.0 & 4.5 \\
\hline Totals/Average & 278 & 79.6 & 3.5 \\
\hline
\end{tabular}

Über 90\% dieser Baurohstoffe kommen aus natürlich vorkommenden Lagerstätten, während die restlichen $10 \%$ aus Recycling-Materialien, marinen Rohstoffe etc. kommt. Die sekundäre Produktion wird weiter steigen, aber im längerfristigen Sinne müssen $85 \%$ der Nachfrage noch von primären Baurohstoffen kommen. Deren Abbau sollte in der Nähe zu den Verbrauchszentren stattfinden aufgrund offensichtlichen wirtschaftlichen und ökologischen Gründen (Leoben, 2010).

Abbildung 1.1 zeigt die Beziehung des Bruttoinlandsprodukts (BIP) pro Kopf für jedes Land in Europa mit entsprechendem Gesamtverbrauch pro Kopf. Deutlich erhöht sich der Konsum von Baurohstoffen mit dem BIP: Es wird erwartet, dass in den kommenden Jahren in den SEE-Ländern sich der Bedarf mit steigendem BIP erhöht. 


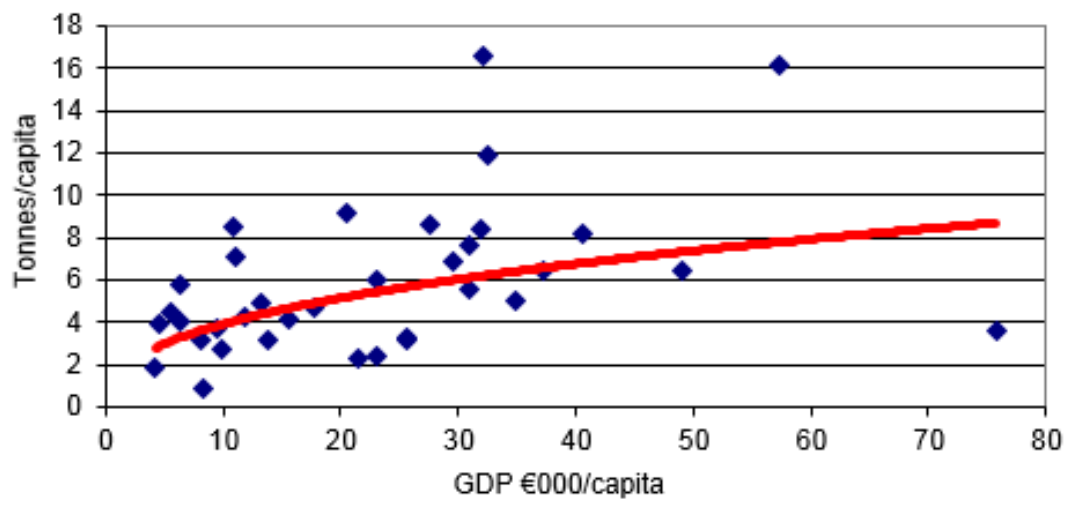

Figure 1.1: GDP per capita versus Tonnes per capita for all European countries (UEPG 2013)

\subsection{Rahmen und Struktur des Handbuches}

Dieses Handbuch konzentriert sich auf die Daten und Methoden, die benötigt werden, um eine nachhaltige Planung von Baurohstoffen zu erreichen, sowie das einen Daten-Rahmen für effektives Ressourcenmanagement für primäre und sekundäre Baurohstoffen unterstützt. Abbildung 1.2 stellt eine vereinfachte Darstellung einer Angebots- und Nachfrage Kette dar zur nachhaltigen Baurohstoff-Planung. Die Planung für Baurohstoffe Versorgung obliegt dem Staat. Sas Ziel ist, die entsprechende Politik, Rechtsrahmen, Aktionen und Informationen zu definieren, die benötigt werden, um die Verfügbarkeit von ausreichenden Mengen von primären und sekundären Baurohstoffe die für die nationalen und regionale Wirtschaft, mittel-und langfristig benötigt werden, zu gewährleisten.

Einige allgemeine Überlegungen werden in Kapitel 2 vorgestellt und das Konzept einer Informationsmatrix ist in Kapitel 3. Kapitel 4 konzentriert sich auf die Datensätze, während Kapitel 5 Datenanalyse Methoden zur Abschätzung der gesamtwirtschaftlichen Nachfrage diskutiert. 


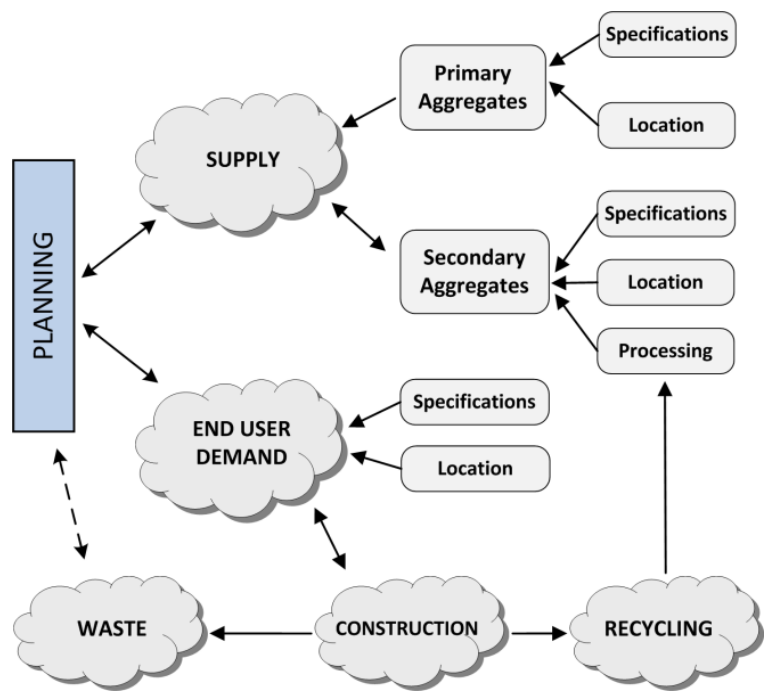

Abb. 1.2: Die Planung sollte die Angebots- und Nachfragekette und andere Einschränkungen betrachten

\subsection{Baurohstoff-Kategorien}

Primäre Baurohstoffe bilden die Produkte der Gewinnung der nicht erneuerbaren natürlichen Ressourcen. Eine rationelle Nutzung ist wichtig. Einer der Wege, um die-

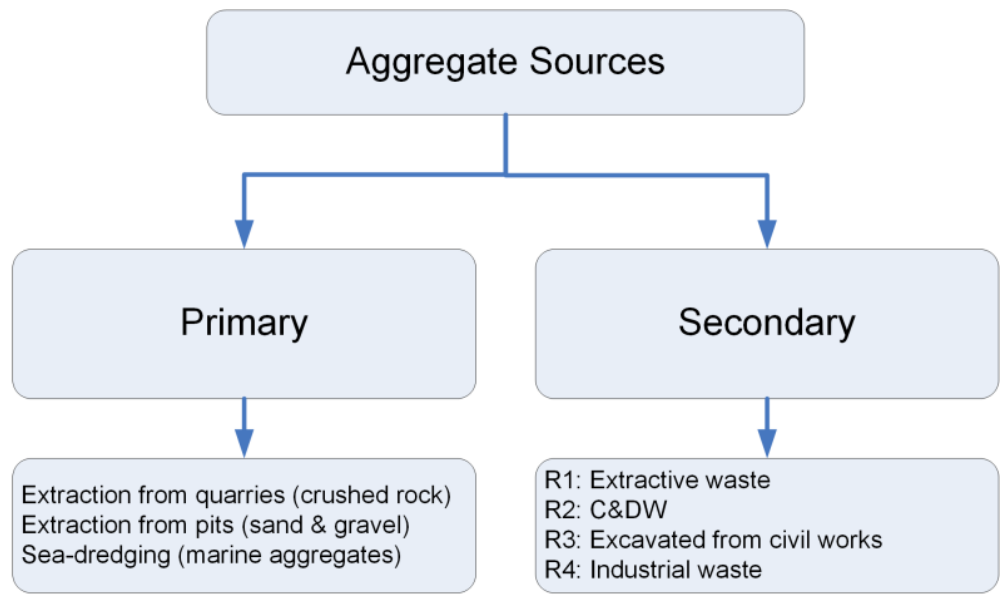

Figure 1.3: Klassfizierung unterschiedlicher Kategorien 
ses Ziel zu erreichen ist, den Markt mit alternativen Materialien liefern. Rezyklierte Gesteinskörnungen können helfen, die zukünftige gesamtwirtschaftliche Nachfrage zu reduzieren durch die Wiederverwendung des Materials, das sonst entsorgt werden würden. 


\section{Bedeutung der Daten und Analyseverfahren für Planung}

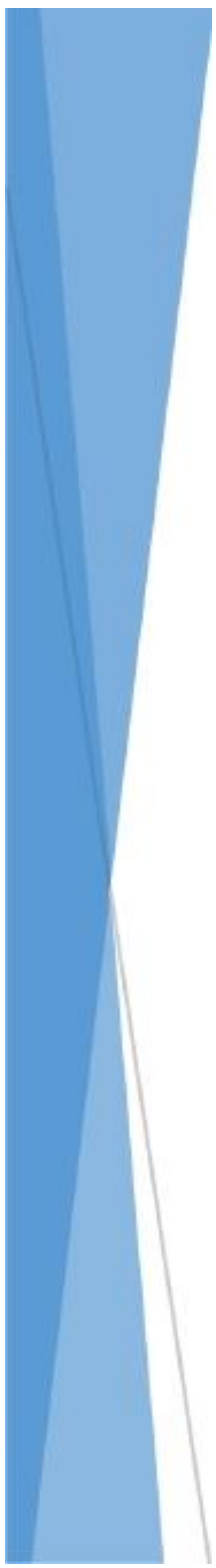

Die Verfügbarkeit von zuverlässigen historischen Datensätzen ist ein wichtiger Beitrag zu einer erfolgreichen Planung. Planungsbehörden auf allen Ebenen sollten Zugang zu Informationen haben wie Reserven, jährliche Produktion von primären und sekundären Baurohstoffe (wo verfügbar im Gesamtversorgung Mix), um in der Lage sein, um die aktuellen Bedürfnisse der Bauwirtschaft erfolgreich zu decken und für künftige Großzivilinfrastrukturarbeiten wie Straßen-und Brückenbau zu planen. 


\subsection{Generelle Berücksichtigung}

Die Planung für Baurohstoffe erfolgt auf nationaler oder regionaler / lokaler Ebene, dies ist abhängig vom Grad der Dezentralisierung eines Lades. Wenn der Planungsprozess noch nicht etabliert ist, was der Fall mit den meisten SEE-Ländern ist, dann ist eine Reihe von vorbereitenden Schritte erforderlich (Bild 2.1).

\subsection{Bedeutung der Daten für Nachhaltiges Planen}

In einfachen Worten, bei der Planung für Baurohstoffe müssen zwei Fragen klar sein: (a) Wie viel Zuschlagmaterial wird benötigt, um die Bedürfnisse eines bestimmten Konsumbereich für einen bestimmten zukünftigen Zeitperiode abzudecken und (b) wie viel ist verfügbar an primären und sekundären Quellen in diesem Bereich?

Es ist selbstverständlich, dass eine große Menge an unterschiedlichen Daten, wie Karten, Zahlen, Statistiken, Gesetze, benötigt wird. Die erforderlichen Daten zu erhalten ist nur ein Teil der Anstrengungen, die Planungsbehörden sollten noch einen Plan für Zuschlagstoffe zu kompilieren. Der andere Teil umfasst alle notwendigen Maßnahmen zum Datenmanagement, dh, die Validierung von Daten, Analysen und Zusammenstellung von Rohdaten in Indizes und neue Daten usw. Darüber hinaus ist

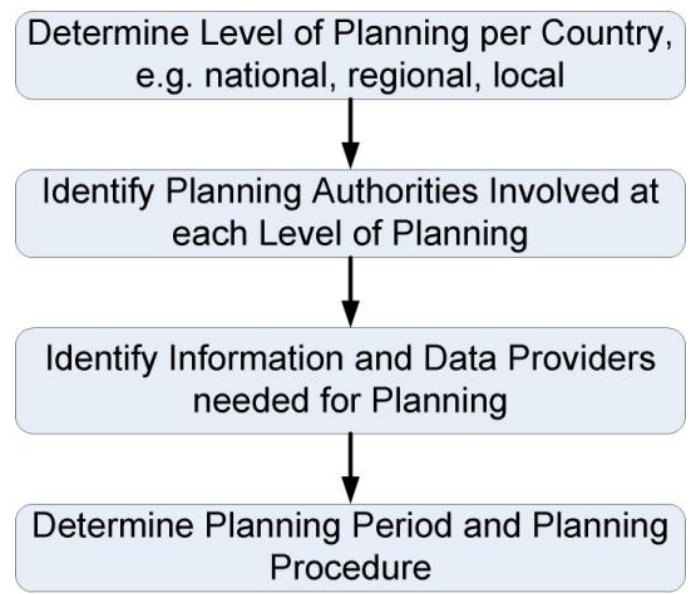

Abb. 2.1: Erforderliche Vorbereitungsschritte für eine nachhaltige Gesamtplanung 


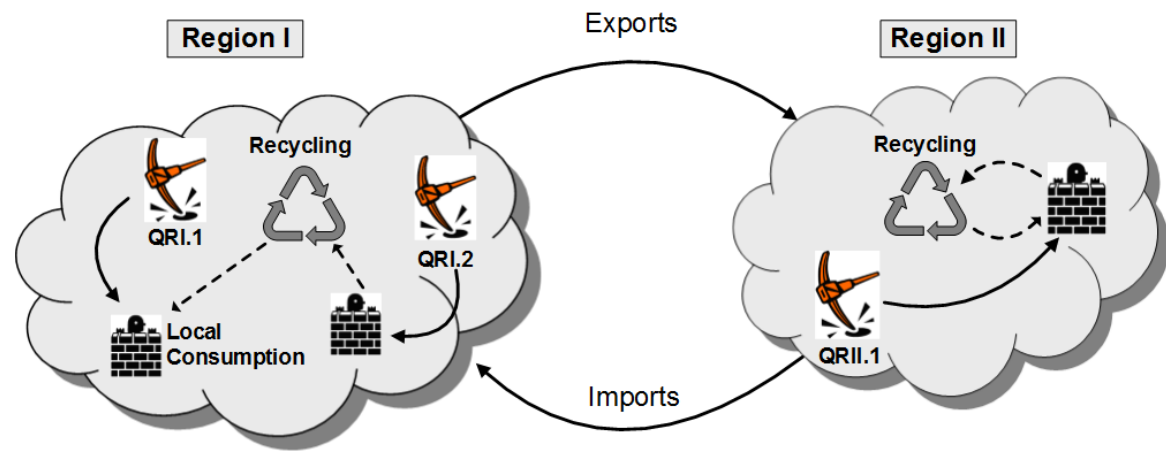

Abb. 2.2: Ein typisches Beispiel für Materialfluss zwischen benachbarten Regionen

eine nachhaltige Planung von Baurohstoffen erfordert Engagement von Methoden wie Lebenszyklus -Analyse, um zu bewerten das ist die bevorzugte Lösung unter Alternativen. Die Notwendigkeit für eine integrale Kenntnis der einschlägigen Bestimmungen, sowohl diejenigen, die vorherrschenden sowie die in Vorbereitung, sollte hinzugefügt werden, die eine Voraussetzung für eine realistische Planungspolitik und / oder Aktivität ist.

\subsection{Datenverfügbarkeit in den SEE-Ländern / Regionen}

Um die verfügbaren Daten für die Planung für primäre und sekundäre Baurohstoffe (zB mineralische Abfälle, Bauschutt und Abbruchmaterial (C \& DW), Industrieabfälle, Material von Bauarbeiten, usw. ausgegraben) für jedes Land / Region, in diesem SEEProjekt zu identifizieren, wurde ein Fragebogen entwickelt. Die Partner des SNAPSEE-Projekt wurden gefragt nach Informationen wie z.B.:

$\checkmark$ wichtige Daten für die Baurohstoffe Planung;

$\checkmark$ Daten, die fehlen oder derzeit nicht in der Planung berücksichtigt sind;

$\checkmark \quad$ Grad der Datenverfügbarkeit und Genauigkeit und der Probleme, die Einfluss auf die Datenerhebung und Genauigkeit;

$\checkmark \quad$ verantwortliche Agentur für die Datenerfassung, Speicherung, Berichterstellung und Aktualisierung;

$\checkmark$ Format, in dem die Daten verfügbar sind; 
$\checkmark$ Detaillierungsgrad der Datenerhebung und deren Quellen;

$\checkmark \quad$ Methoden der Datenanalyse, die verwendet werden, um Rohdaten in nützliche ergänzende Informationen zu Baurohstoffe Planung umzuwandeln.

Die grundlegenden geologischen Informationen zur Verteilung der primären Gesamtressourcen (zB geologische Karten von 1: 50.000 bis 1: 100.000, Lagepläne der Abbaustätten und Altlasten, Lithologien, eine breit angelegte Bewertung der Qualität der Ressourcen, etc.) werden in der Regel durch nationale geologische Untersuchung des Landes zur Verfügung gestellt.

Zusammenfassung der wichtigsten Ergebnisse der Umfrage: es wurde der Schluss gezogen, dass i) die Vorräte sind nicht gleichmäßig in allen entwickelten SEE-Ländern / Regionen, ii) die in diesen Aufstellungen enthaltenen Daten werden nicht regelmäBig aktualisiert oder in digitaler Form aufbewahrt, iii) die Daten können aus unterschiedlichen Quellen stammen, und deshalb sind sie nicht immer kompatibel und leicht nachvollziehbar, iv) die in den Vorräten registrierten Informationen kann daher nicht regelmäßig gegengeprüft werden, Probleme mit der Zuverlässigkeit. 
Table 2.1: Verfügbarkeit von Daten sekundärer Ressourcen in den SEE-Ländern (Hatzilazaridou, 2014)

\begin{tabular}{|c|c|c|c|c|c|c|}
\hline \multirow[b]{2}{*}{$\begin{array}{l}\text { Coun- } \\
\text { try/Region }\end{array}$} & \multicolumn{4}{|c|}{$\begin{array}{c}\text { Data on production volumes per type of re- } \\
\text { cycled waste (tonnes) }\end{array}$} & \multirow[b]{2}{*}{$\begin{array}{c}\text { Data on \% } \\
\text { of second- } \\
\text { ary mate- } \\
\text { rials re- } \\
\text { cycled }\end{array}$} & \multirow[b]{2}{*}{$\begin{array}{c}\text { Data on } \\
\% \text { of re- } \\
\text { cycled } \\
\text { used as } \\
\text { aggre- } \\
\text { gates }\end{array}$} \\
\hline & $\mathbf{R} 1\left(^{*}\right)$ & $\mathbf{R} 2\left(^{*}\right)$ & $\mathbf{R} 3\left(^{*}\right)$ & R4(*) & & \\
\hline Albania & $\begin{array}{c}\text { Esti- } \\
\text { mated }\end{array}$ & NO & NO & Estimated & NO & NO \\
\hline Austria & $\begin{array}{l}\text { Esti- } \\
\text { mated }\end{array}$ & YES & NO & YES & $\begin{array}{l}\text { YES (R2) } \\
\text { YES (R4) }\end{array}$ & NO \\
\hline Croatia & $\begin{array}{c}\text { Esti- } \\
\text { mated }\end{array}$ & Estimated & $\begin{array}{c}\text { Esti- } \\
\text { mated }\end{array}$ & Estimated & NO & NO \\
\hline $\begin{array}{l}\text { Emilia Ro- } \\
\text { magna }\end{array}$ & YES & YES & NO & YES & YES & NO \\
\hline Greece & $\mathrm{NO}$ & Estimated & $\mathrm{NO}$ & Estimated & $\mathrm{NO}$ & NO \\
\hline $\begin{array}{l}\text { Herzegbos- } \\
\text { nian Canton }\end{array}$ & NO & NO & NO & NO & NO & NO \\
\hline Hungary & $\begin{array}{l}\text { Esti- } \\
\text { mated }\end{array}$ & Estimated & NO & Estimated & YES & NO \\
\hline $\begin{array}{l}\text { Montene- } \\
\text { gro }\end{array}$ & $\begin{array}{l}\text { Esti- } \\
\text { mated }\end{array}$ & NO & NO & Estimated & NO & NO \\
\hline Romania & $\begin{array}{c}\text { Esti- } \\
\text { mated }\end{array}$ & NO & NO & Estimated & NO & NO \\
\hline Serbia & $\begin{array}{c}\text { Esti- } \\
\text { mated }\end{array}$ & NO & NO & Estimated & $\begin{array}{l}\text { YES (R1) } \\
\text { NO (R4) }\end{array}$ & $\begin{array}{l}\text { YES (R1) } \\
\text { NO (R4) }\end{array}$ \\
\hline Slovakia & $\begin{array}{l}\text { Esti- } \\
\text { mated }\end{array}$ & Estimated & NO & Estimated & $\begin{array}{l}\text { NO(R1) } \\
\text { YES (R2) } \\
\text { YES( R4) }\end{array}$ & NO \\
\hline Slovenia & $\begin{array}{l}\text { Esti- } \\
\text { mated }\end{array}$ & YES & NO & Estimated & $\begin{array}{l}\text { YES( R1) } \\
\text { YES( R2) } \\
\text { NO (R4) }\end{array}$ & NO \\
\hline Trento & $\begin{array}{l}\text { Esti- } \\
\text { mated }\end{array}$ & YES & $\begin{array}{l}\text { Esti- } \\
\text { mated }\end{array}$ & YES & $\begin{array}{l}\text { NO (R1) } \\
\text { YES (R2) } \\
\text { NO (R3) } \\
\text { YES (R4) }\end{array}$ & YES (R2) \\
\hline
\end{tabular}

$\left(^{*}\right)$ See Fig 1.2 for explanation 


\section{Organisieren der Informationen}

Baurohstoff-Planung beinhaltet die Analyse von unterschiedlichen Prozessen im Lebenszyklus von Materialien. Diese Prozesse sind als "Schwerpunkte" bezeichnet; die Planung sollten sie individuell und kollektiv zugleich ansprechen. Solche Prozesse sind mit Stoffströme, die pro Planungsebene variieren miteinander verbunden. Die Informationsmatrix stellt eine hierarchische Struktur dar, die für jeden Planungs-Bereich benötigten Daten werden explizit gekennzeichnet. Eine Informationsmatrix sollte für jede Planungsebene festgelegt werden. 
Baurohstoff-Planung beinhaltet die Analyse von unterschiedlichen Prozessen im Lebenszyklus von Materialien. Diese Prozesse sind als "Schwerpunkte" bezeichnet; die Planung sollten sie individuell und kollektiv zugleich ansprechen. Solche Prozesse sind mit Stoffströme, die pro Planungsebene variieren miteinander verbunden. Die Informationsmatrix stellt eine hierarchische Struktur dar, die für jeden Planungs-Bereich benötigten Daten werden explizit gekennzeichnet. Eine Informationsmatrix sollte für jede Planungsebene festgelegt werden.

\subsection{Planungskomponenten}

Nachhaltige Baurohstoff-Planung stellt eine komplizierte Frage dar. Abbildung 3.1 zeigt eine Konzeptdarstellung, worum es geht, eine fundierte Planungsentscheidung zu erreichen. Rohdaten aus einer Vielzahl von Quellen werden auf verschiedene Weise analysiert, überprüft, für die Analyseberechnungen verwendet und schließlich in den Planungsprozess implementiert. Es gibt mehrere Rückkopplungsverbindungen zwischen den verschiedenen Prozessen und Stufen wie in einem komplizierten System erwartet wird. Ein Schema zur besseren Organisation dieses Informationsfluss unter Verwendung des Konzepts der Informationsmatrix wird dargestellt.

\subsection{Einrichten der Informationsmatrix}

Der Zweck dieses Handbuchs ist es, den Planungsbehörden der SEE-Ländern zu helfen, eine effektive Verwaltung der für die Planung notwendigen Daten aufzubauen. Dies kann komplex sein, aufgrund: i) die Heterogenität, die in SEE-Ländern für die Datenverwaltung existiert; ii) das mögliche Fehlen von Werkzeugen für die Unterstützung und Regelung der Baurohstoffe Entwicklung in einigen dieser Länder; iii) die Komplexität in den bestehenden Mechanismen der Datenerhebung, die oft aufgefallen ist; iv) das Fehlen von Daten und Information mit hoher Genauigkeit, sowie der Mangel an für die Datenvalidierung geeigneten Methoden.

Unter Berücksichtigung der oben beschriebenen Situation, wird vorgeschlagen, dass einige Grundprinzipien festgelegt werden sollten und anschließend für Daten und Analysen die Methoden für die Baurohstoffe Planung, die von den Planungsbehörden aller SEE-Ländern verständlich sein wird. 
1. Zuverlässige Informationen ist die solide Basis für eine erfolgreiche Planung;

2. Baurohstoffe-Produktion, Verwendung und Recycling beinhalten eine Reihe von separaten Aktivitäten, die nachhaltige Planung sollte diese Aktivitäten berücksichtigen. Dies bedeutet, dass eine Vielzahl von unterschiedlichen Arten von Daten benötigt werden;

3. Es ist wichtig, eine Strategie für die Sammlung und eine Routine für die Organisation der oben genannten Daten zu entwickeln;

Bevor Sie einen Planungsprozess auf jeder Ebene initiieren, sollte die (große Menge) Informationen zu dieser Planungsebene eindeutig identifiziert werden. Es wurde ein Werkzeug geschaffen, um diese Informationen zu organisieren, um die Identifizierung der Gesamtströme zwischen mehreren Prozessen zu erleichtern (oder konzentrieren Bereichen) wie Primärproduktion, Recycling, usw.

Die Informationen werden in Form einer Matrix vorgesehen in mindestens vier Spalten und eine variable Anzahl von Zeilen. Jede Zeile stellt einen Prozess oder eine Phase der Kette dar, während die Spalten die Felder definieren, die die Informationen in jeder Zeile charakterisieren. Sobald die Informationen Matrix zu einer bestimmten Planungsebene abgeschlossen ist, wird dann die Datenvalidierung und Datenanalyse eine einfache Aufgabe. Abbildung 3.2 stellt eine vereinfachte Informationsstruktur mit sieben (7) Schwerpunktbereiche oder Prozesse und vier (4) Spalten mit Informationen. 


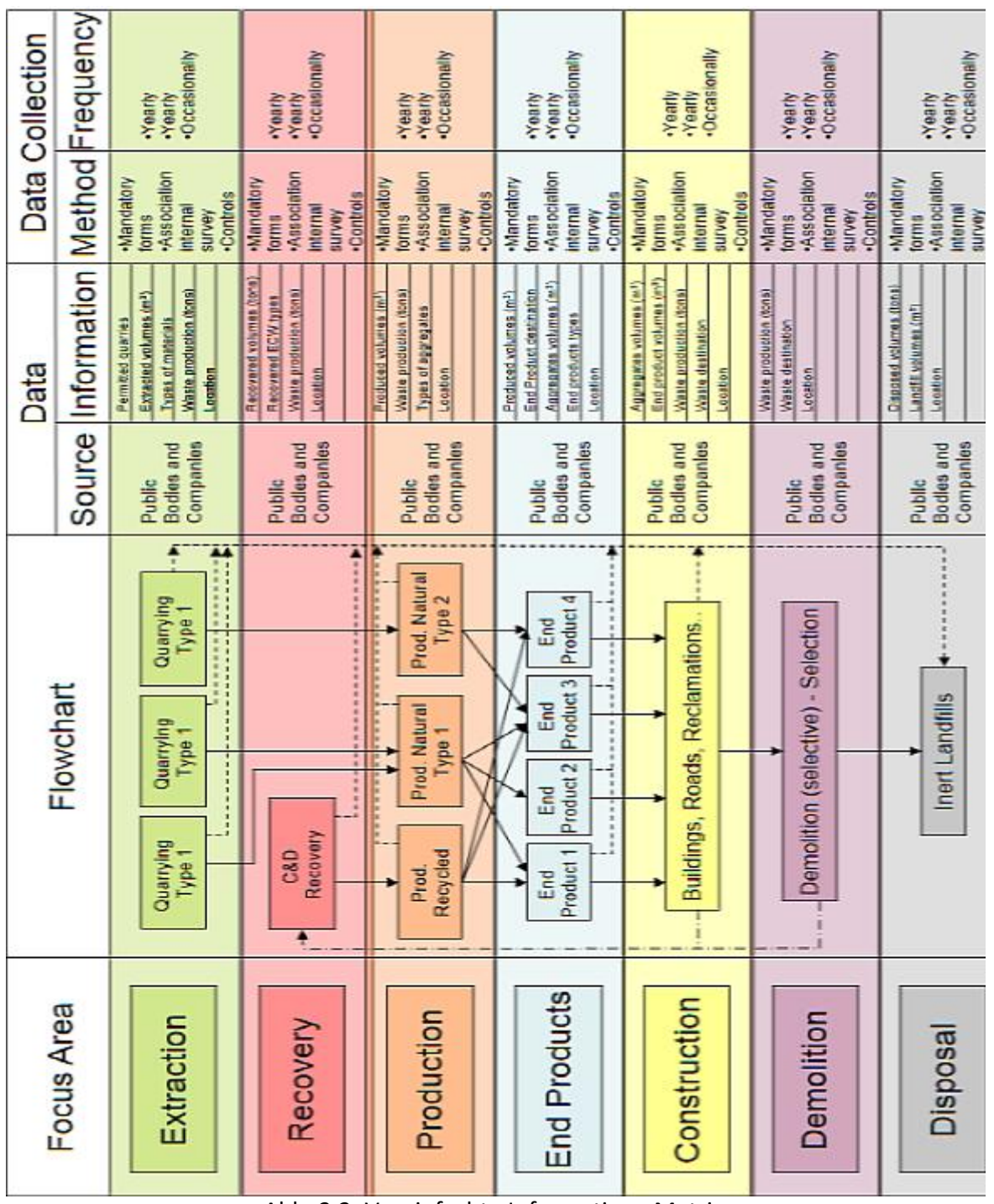

Abb. 3.2: Vereinfachte Informations-Matrix 


\subsection{Begrenzungen}

Die Planungsbehörden haben klar definierte geographischen Grenzen. Diese Grenzen, die Regionen oder Nationen zu entsprechen, sollte in der Datenanalyse berücksichtigt werden. Zudem wäre es sehr interessant, dieses Konzept in die Nachbarregionen mit gemeinsamen geografischen oder anderen Merkmalen zu verbinden. Jede Information oder Datenbankschema ist so gut wie die Qualität der Daten, die es zu verwalten gilt. 


\section{Ermittlung des Datenbedarfs}

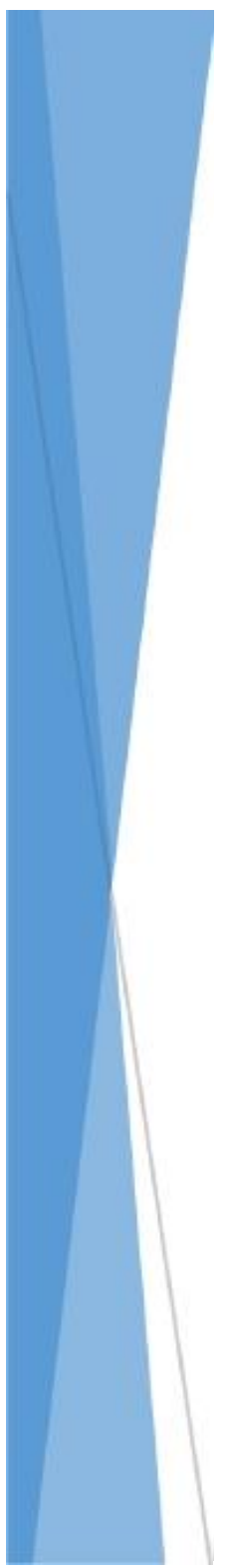

Die Bereitstellung und Nutzung von regelmäßigen, zuverlässigen und aktuellen Daten ist von größter Bedeutung. Die Schaffung effizienter Verfahren der Datenerhebung,-Management und Datenverarbeitung sind für die Entwicklung von harmonisierten SARM (Sustainable Aggregates Resource Management) und SSM (Sustainable Supply Mix) Politik notwendig. Daten nützlicher Informationen, die in einer leichter zugänglichen Form für die Verwendung durch die politischen Entscheidungsträger und Entscheidungsgremien sowie der Öffentlichkeit verarbeitet werden können. Nachhaltige Baurohstoff-Planung hängt ab von zuverlässigen Daten. Gleichzeitig haben die Planungsbehörden nicht immer die Ressourcen und die Fähigkeit, grundlegende Daten zu sammeln, so dass in vielen Fällen auf die Überwachung und Datensammlung Bemühungen von anderen Agenturen abgestellt wird. In jedem Fall sollte die Entscheidungsfindung auf den besten verfügbaren, wissenschaftlich fundierte Daten aus allgemein anerkannten Quellen basieren. "Perfekte" Daten sind nicht immer notwendig oder möglich, aber die Daten "Qualität" sollte ausreichend sein. Daten Schätzungen kann mit Vorsicht verwendet werden, wenn aktuelle Daten sind nicht verfügbar sind. 


\subsection{Datenformat}

Daten können auf nationaler und regionaler Ebene oder je nach dem Niveau, das die Planung angelegt wird, gesammelt werden. Zwar können die für die jeweilige Aufgabe benötigten Daten unterschiedlich sein, sie können im allgemeinen wie folgt kategorisiert werden:

$>$ statistische Grundlagen;

$>$ Karten der verschiedenen Skalen und Fernerkundungsdaten;

$>$ thematischen Informationen wie Netzwerke, Infrastruktur, etc.

$>$ Es gibt Daten in einer Vielzahl von Formen d.h. Daten von

- wissenschaftlichen Artikel, Berichte, Dokumente, Tabellen, Diagrammen und Karten in verschiedenen Maßstäben;

- Satellitenbildern, Luftaufnahmen oder andere Formen der visuellen Daten.

\subsection{Identifizierung der wichtigsten Datensätze für Primärbauroh- stoffe}

Daten müssen die folgenden Angaben enthalten:

$>$ Daten für die geologische Verteilung und Qualität der Ressourcen;

$>$ Daten, die das aktuelle Ausmaß der zulässigen Reserven zu beschreiben, die Rate der Erschöpfung durch Verkäufe, die Rate des Ersatzes durch neue Berechtigungen;

$>$ Daten für Gesamt Vertrieb, Produktion / Produktionskapazität, Verbrauch und Import / Export, in der Regel ein Ergebnis von Umfragen;

$>$ Verzeichnis der aktiven und inaktiven (erlaubt) Abbaue;

$>$ Daten über den Betrieb von Abbaue; 
D Daten zu den Gesamttransport von der Entnahmestelle zu Verbrauchermärkten ;

$>$ Daten über die Einhaltung der gesamtwirtschaftlichen Ressourcen mit technischen Spezifikationen;

> Daten für die Gesamtnachfrage; Ergebnis der Bedarfsprognosen aufgund von Szenarien geeigneter Methoden.

Die Bedeutung von jedem der obigen Datensätze wird nachfolgend kurz beschrieben.

\subsubsection{Daten für die Sicherung mineralischer Baurohstoffe in Raumnutzungsplänen}

"Raumnutzungspläne enthalten in der Regel Kriterien welche Art von Entwicklung in bestimmten Bereichen erfolgen und sind ein typisches Beispiel für Pläne, die den Rahmen für die künftige Genehmigung setzt" (SEA, 2001). Entscheidungsfindung für Landnutzungszuweisung wird auch auf Umweltaspekte beruhen; Beispiele sind $\mathrm{Na}$ turschutzgebiete und Korridore für die Migration der Arten, Flussgebietsmanagement und Hochwasserschutz, und Boden- und Wasserschutz. Politischen Entscheidungen, die Landnutzung definieren, werden vor allem durch die Raumplanung und die damit verbundenen funktionellen Zonierung des Landes realisiert. Dies beinhaltet Kompromisse zwischen vielen sektoralen Interessen, einschließlich der Industrie, Transport, Kommunikation, Bergbau, Landwirtschaft und Forstwirtschaft.

Eine nachhaltige Baurohstoffe-Politik muss Baurohstoffe Vorkommen auf Basis der Raumnutzungsplanung sichern, um weitere Konflikte zu vermeiden. Dabei sollten Informationen über die Bedeutung der identifizierten Ressourcen auf nationaler, regionaler und lokaler Ebene vorhanden sein. Die Raumordnungspolitik für Baurohstoffe sollte gut strukturierte und zuverlässige Daten nützen auf der Grundlage:

$>$ einer digitale geologische Wissensbasis (geologische Karten 1: $50.000 \mathrm{Zu}$ sätzliche geologische Vermessungen könnte benötigt werden im Maßstab: 25.000 bis $1: 10.000$.);

$>$ eine transparente Methodik zur Identifizierung der verfügbaren Gesamtquellen (Qualität, Quantität, lokaler Bedeutung);

langfristig Schätzungen für die regionale und lokale Nachfrage für Zuschlagstoffe, unter Berücksichtigung anderer Quellen (zB sekundäre Gesamtressourcen ). 
Zwei Beispiele werden empfohlen:

$>$ Der österreichische Rohstoffplan (2010), der als "best practice" Beispiel für eine nationale Landnutzungspolitik (Österreich, 2014) gewählt wurde;

$>$ Ansatz des British Geological Survey (BGS 2012).

\subsubsection{Daten um die Wissensbasis der geologischen Verteilung geeigneter primärer Ressourcen zu verbessern}

Die Menge der Baurohstoffe, die langfristig benötigt werden, hängt von Lage der Wirtschaft und der daraus resultierenden Bautätigkeit sowie der künftigen Verfügbarkeit von Ressourcen für Investitionen ab. Wissen über Gesamtvermögen ist von wesentlicher Bedeutung für die Herstellung von wirksamen und nachhaltigen Planungsentscheidungen. Effizienz und Effektivität des Planungssystems ist abhängig von hoher Qualität, leicht zugängliche Informationen über den Umfang, die Qualität und, wenn möglich, die Menge der Mineralressourcen und ihre Beziehung zu den nationalen Planungsvorhaben, die Einschränkungen für die Gewinnung von Mineralrohstoffen darstellen könnten.

Ein Hauptziel ist es, dass Basisdaten in einem einheitlichen Format, die aktualisiert werden können, überarbeitet und angepasst werden, um den Planungsbedürfnissen Rechnung zu tragen. Dies erfordert Verständnis für die Lage, Umfang, Art und Zugänglichkeit der Ressource. Ansätze, um Baurohstoff-Ressourcen zu identifizieren, unterscheiden sich im Detailgrad und Umfang je nach den Bedürfnissen, und die Verfügbarkeit in einem Gebiet. Ein gemeinsamer Ansatz ist es, die Gesamtressourcen in seinen geologischen Komponenten und Skala (empfohlen Karte im Maßstab 1: 50.000) zu identifizieren. An diesen Maßstäben und in Ermangelung anderer Daten aus Extraktionsstellen, und systematische Stichproben, können nur Informationen über die Flächenausdehnung der Ressourcen bereitgestellt werden. National Geological Surveys sind in der Regel die Daten Anbieter von solchen Informationen, dh bez. Qualität und Quantität der Gesamtressourcen .

Identifizierung der strategischen Gesamt Ressourcen sollten auch andere wichtige Faktoren identifizieren, die den Zugang zu den Ressourcen beeinflussen können. Deshalb ist es wichtig, dass die regionale / lokale Planungsbehörden Informationensammeln und alle verschiedenen Informationsebenen auf einer Karte berücksichtigen (empfohlen Maßstab 1: 100.000), mit dem Einsatz von GIS-Tools. Vier Hauptelemente der Informationen könnten dargestellt werden: 
$>$ die geologische Verteilung aller Onshore-Aggregat Ressourcen und die Location des Gesamtabbaustätten

$>$ das Ausmaß der Genehmigungen

$>$ das Ausmaß der Naturschutz-Bezeichnungen

Die Auswirkungen der genannten Einschränkungen in Bezug auf den Zugang zu Ressourcen und die Gesamtversorgung der Qualitätszuschlagsstoffen sollten in regelmäßigen Abständen (zB alle 5 Jahre) durch den jeweiligen öffentlichen Verwaltung befragt, in Zusammenarbeit mit einer Reihe von Interessengruppen (zB Industrie, NGOs, Kommunen und Behörden) und bei Bedarf Korrekturmaßnahmen und alternative Optionen angenommen werden sollte.

\subsubsection{Daten zu Produktion / Produktionskapazität, Verbrauch und Import / Export}

Diese Erhebungen (kompiliert alle 4 Jahres-Abständen) sollte vertiefende und aktuelle Informationen bieten zu den nationalen und regionalen Vertrieb, interregionale Ströme, Transport, Produktion und Verbrauch und bewilligten Reserven von PrimärBaurohstoffen. Diese Daten werden in der Regel aus offiziellen Quellen (zB Planungsbehörden) und Betriebsgesellschaften geliefert und sollten regelmäßig aktualisiert werden. Maßeinheiten sind Tonnen. Werte sollten für Import / Export und Vertrieb zur Verfügung gestellt werden.

\subsubsection{Informationen}

Eine Datenbank der aktiven und inaktiven Steinbrüche sollten Informationen über halten:

$>$ Status des Steinbruchs (aktiv oder inaktiv);

$>$ Name des Steinbruchs

$>$ geographische Lage (Koordinaten);

Steinbruch-Adresse;

$>$ Betreiber;

> Geologie (basierend auf einer 1: 50.000 geologische Karte); 
Produktionszahlen pro Produkt (in Tonnen);

$>$ Produktionskapazität (in Tonnen);

$>$ Produkt End-Anwendungen, bei denen bekannt ist;

$>$ Absatzmenge;

$>$ Ablaufdatum der Genehmigung;

$>$ gesamten Ressourcen / Reserven;

$>$ verbleibenden Ressourcen / Reserven;

$>$ Produktionszahlen für die vergangenen 3 Jahre (gilt für vorübergehend inaktiv Steinbrüche).

Die Datenbank und die zugehörigen GIS Datensatz wird ein wertvolles Instrument für die Überwachung der unter anderem die Produktionskapazität, Reserven und Ressourcenverbrauch bieten.

\subsubsection{Daten, um die Auswirkungen des Transports von Zuschlagstoffen aus der Abbaustätte zu den Verbrauchermärkten zu bewerten}

Baurohstoffe werden in der Regel auf der Straße nicht mehr als $50 \mathrm{~km}$ transportiert. Dabei sollte ein Konzept über den Abtransport der Rohstoffe von den Abbauen, das nach von der Standortgemeinde bzw. unter Umständen auch von Nachbargemeinden bekannt gegebenen Verkehrsgrundsätzen (Routenwahl, Transportgewicht, Transportzeiten u. dgl.) ausgearbeitet werden.

\subsubsection{Daten über die Einhaltung der gesamtwirtschaftlichen Ressourcen mit tech- nischen Spezifikationen}

Alle Baurohstoffe (primär und sekundär) müssen bestimmte vorgeschriebene Mindest-Standards erfüllen, wenn sie in der Bauwirtschaft verwendet werden. Sie sollten für einen bestimmten Zweck unter einer Bescheinigung der Leistung passt, oder sollte mit den einschlägigen nationalen oder EU-Normen entsprechen. Um sicherzustellen, dass Baurohstoffe kontinuierlich die erforderliche Spezifikation erfüllen, ist das Endprodukt für die beabsichtigte Verwendung aus einer Reihe von Labortests entwickelt worden. Eine vollständige Liste der von diesen neuen europäischen Normen etablierten Tests sind unten angegeben. Die entsprechende Norm Dokument sollte 
konsultiert, wenn die vollständigen Details der Tests und Methoden benötigt werden.

EN 932 Prüfverfahren für allgemeine Eigenschaften von Gesteinskörnungen

EN 933 Prüfverfahren für geometrische Eigenschaften von Gesteinskörnungen

EN 1097 Prüfverfahren für mechanische und physikalische Eigenschaften von Gesteinskörnungen

EN 1367 Prüfverfahren für thermische Eigenschaften und Verwitterungsbeständigkeit von Gesteinskörnungen

EN 1744 Prüfverfahren für chemische Eigenschaften von Gesteinskörnungen

Es gibt auch europäische Standards, die den Bereich der akzeptablen Werte unter jedem Test angeben. Baurohstoffe sollten in diese Bereiche fallen, um als geeignet für diese Verwendung werden. Die relevanten Normen sind:

EN 12620 Gesteinskörnungen für Beton

EN 13043 Gesteinskörnungen für Asphalt und Oberflächenbehandlungen

EN 13055 Leichte Gesteinskörnungen

EN 13139 Gesteinskörnungen für Mörtel

EN 13242 Gesteinskörnungen für ungebundene und hydraulisch gebundene Materialien

EN 13383 Armour Stein

EN 13450 Bahn Ballast.

\subsubsection{Daten für Nachfrageprognosen}

Baurohstoffe Nachfrage ist durch Aktivität in der Bauwirtschaft und der Wirtschaft als Ganzes bedingt. Nach UEPG (2013) sind etwa 30 kt Baurohstoffe pro km neuer Straßen nötig. Daher spielen Nachfrageprognosen eine wichtige Rolle in der nationalen und regionalen Politikformulierung. Siewerden verwendet, um mittel-und langfristig Versorgungsanforderungen zu bestimmen (siehe Kapitel 5). 


\subsection{Identifizierung von Hauptdatensätzen für Sekundäre Bauroh- stoffe}

Vier Arten von Recycling-Aktivitäten werden als potentielle Quellen berücksichtigt und diese sind in Tabelle 4.1 dargestellt. Daten, müssen die folgenden Angaben enthalten:

$>$ Daten für technische Spezifikationen;

$>$ Daten für Umweltauswirkungen;

$>$ Volumen und Prozentangaben je nach Art der erzeugten Sekundärressourcen ;

$>$ Volumen und Prozentangaben pro Art von Recycling-Sekundärressourcen ;

$>$ Volumen und Prozentangaben von Recyclingmaterial als Baurohstoffe.

Die gleichen europäischen Standards, die Primär Baurohstoffe (siehe Abschnitt 4.3.8) gelten auch für die Neben Baurohstoffe Ressourcen gelten. Die Standards werden auf der Baurohstoffe Tauglichkeit eher als die Quelle.

Es werden einige Beispiele erwähnt (in Englisch).

\section{Geological Resources}

\begin{tabular}{|l|l|l|l|l|l|l|}
\hline $\begin{array}{l}\text { Administra- } \\
\text { tive Planning } \\
\text { District (i.e., } \\
\text { Region, State) }\end{array}$ & $\begin{array}{l}\text { Location } \\
\text { Coordi- } \\
\text { nates }\end{array}$ & $\begin{array}{l}\text { Formation } \\
\text { Type }\end{array}$ & $\begin{array}{l}\text { Map } \\
\text { Scale }\end{array}$ & Reserves & $\begin{array}{l}\text { Estimate of } \\
\text { Technical / } \\
\text { Quality Cha- } \\
\text { racteristics }\end{array}$ & $\begin{array}{l}\text { Respon- } \\
\text { sible } \\
\text { agency }\end{array}$ \\
\hline & & & & & & \\
\hline
\end{tabular}

\section{Geological Reserves}

\begin{tabular}{|l|l|l|l|l|l|}
\hline $\begin{array}{l}\text { Administrative } \\
\text { Planning District } \\
\text { (e.g., Region, } \\
\text { State) }\end{array}$ & $\begin{array}{l}\text { Location } \\
\text { Coordi- } \\
\text { nates }\end{array}$ & $\begin{array}{l}\text { Formation } \\
\text { Type }\end{array}$ & Map Scale & Reserves & $\begin{array}{l}\text { Technical } \\
\text { / Quality } \\
\text { Characte- } \\
\text { ristics }\end{array}$ \\
\hline $\begin{array}{l}\text { e.g., Resource } \\
\text { Zone, Quarry, etc. }\end{array}$ & & & & & \\
\hline
\end{tabular}

\section{Permitted Reserves}




\begin{tabular}{|l|l|l|l|l|l|l|}
\hline $\begin{array}{l}\text { Administrative } \\
\text { Planning District } \\
\text { (i.e., Region, } \\
\text { State) }\end{array}$ & $\begin{array}{l}\text { Location } \\
\text { Coordi- } \\
\text { nates }\end{array}$ & $\begin{array}{l}\text { Formation } \\
\text { Type }\end{array}$ & $\begin{array}{l}\text { Map } \\
\text { Scale }\end{array}$ & Reserves & $\begin{array}{l}\text { Technical } \\
\text { / Quality } \\
\text { Characte- } \\
\text { ristics }\end{array}$ & $\begin{array}{l}\text { Permit } \\
\text { Holder }\end{array}$ \\
\hline $\begin{array}{l}\text { e.g., Resource } \\
\text { Zone, Quarry, etc. }\end{array}$ & & & & & & \\
\hline
\end{tabular}

\section{Primary Aggregates}

\begin{tabular}{|l|l|l|l|l|l|}
\hline $\begin{array}{l}\text { Quarry or Pit } \\
\text { Information } \\
\text { (Active \& } \\
\text { Inactive) }\end{array}$ & $\begin{array}{l}\text { Quarry Loca- } \\
\text { tion (Coordi- } \\
\text { nates) }\end{array}$ & $\begin{array}{l}\text { Year } \\
\text { Start of } \\
\text { Permit }\end{array}$ & $\begin{array}{l}\text { Year } \\
\text { End of } \\
\text { Permit }\end{array}$ & $\begin{array}{l}\text { Type of rock } \\
\text { Formation(s) }\end{array}$ & $\begin{array}{l}\text { Technical Characte- } \\
\text { ristics (\#) }\end{array}$ \\
\hline 1. & & & $\begin{array}{l}\text { e.g., limes- } \\
\text { tone, igne- } \\
\text { ous rock, } \\
\text { etc. }\end{array}$ & $\begin{array}{l}\text { e.g., chemical, mi- } \\
\text { neralogical compo- } \\
\text { sition, Los Angeles } \\
\text { and compressive } \\
\text { strength, etc. }\end{array}$ \\
\hline
\end{tabular}

(\#) per recommended application

\section{Active Quarries for Primary Aggregates}

\begin{tabular}{|l|l|l|l|l|l|}
\hline $\begin{array}{l}\text { Production } \\
\text { Center }\left(^{*}\right)\end{array}$ & $\begin{array}{l}\text { List of } \\
\text { Active } \\
\text { Quarries }\end{array}$ & $\begin{array}{l}\text { Year of } \\
\text { reporting }\end{array}$ & $\begin{array}{l}\text { Annual Pro- } \\
\text { duction } \\
\text { (tonnes) }(* *)\end{array}$ & $\begin{array}{l}\text { Current } \\
\text { Capacity } \\
\text { (tonnes) }\end{array}$ & $\begin{array}{l}\text { Remaining Re- } \\
\text { serves (tonnes) }\end{array}$ \\
\hline \multirow{2}{*}{1} & 1.1 & & & & \\
\cline { 2 - 6 } & 1.2 & & & & \\
\cline { 2 - 6 } & $1 . n$ & & & & \\
\hline 2 & 2.1 & & & & \\
\hline & $2 . n$ & & & & \\
\hline
\end{tabular}

$\left({ }^{*}\right)$ Depends on the Level of Planning, e.g. District, Quarrying area, Region, Municipality, Quarry, etc.

$(* *)$ per produced size

\section{Inactive Quarries for Primary Aggregates}

\begin{tabular}{|l|l|l|l|l|}
\hline $\begin{array}{l}\text { Production } \\
\text { Center }\left({ }^{*}\right)\end{array}$ & $\begin{array}{l}\text { Number of } \\
\text { Inactive Qua- } \\
\text { rries }\end{array}$ & $\begin{array}{l}\text { Year of report- } \\
\text { ing }\end{array}$ & $\begin{array}{l}\text { Current Capaci- } \\
\text { ty (tonnes) }\end{array}$ & $\begin{array}{l}\text { Remaining } \\
\text { Reserves } \\
\text { (tonnes) }\end{array}$ \\
\hline & & & & \\
\hline
\end{tabular}

(*) Depends on the Level of Planning, e.g. District, Region, Municipality, Quarry, etc.

\section{Land Use Planning}

\begin{tabular}{|l|l|l|}
\hline Identification of extraction areas & Location Coordinates & Identity of responsible agency \\
\hline $\begin{array}{l}\text { Areas where extraction is not } \\
\text { allowed }\end{array}$ & & Local/regional Government \\
\hline $\begin{array}{l}\text { Areas where extraction may be } \\
\text { allowed under conditions }\end{array}$ & & \\
\hline
\end{tabular}




\begin{tabular}{|l|l|l|}
\hline Extraction is permitted & & \\
\hline Resources are safeguarded & & \\
\hline
\end{tabular}

\section{Environmental Impacts due to proposed aggregate planning}

\begin{tabular}{|l|l|l|}
\hline $\begin{array}{l}\text { Monitoring of air quality near } \\
\text { operating units }\end{array}$ & & \\
\hline $\begin{array}{l}\text { Monitoring of water quality near } \\
\text { operating units }\end{array}$ & & \\
\hline Other environmental constraints & & \\
\hline & & \\
\hline
\end{tabular}

\section{Secondary Production for Mining / Extractive waste / byproduct / residue (R1)}

\begin{tabular}{|l|l|l|l|l|l|}
\hline $\begin{array}{l}\text { Secondary } \\
\text { Aggregate } \\
\begin{array}{l}\text { Facilities } \\
\text { (quarry, } \\
\text { mine) }\end{array}\end{array}$ & $\begin{array}{l}\text { Facility Location } \\
\text { (Coordinates) }\end{array}$ & $\begin{array}{l}\text { Year } \\
\text { Start of } \\
\text { Permit }\end{array}$ & $\begin{array}{l}\text { Year End } \\
\text { of Permit }\end{array}$ & $\begin{array}{l}\text { Type of } \\
\text { Product(s) }\end{array}$ & $\begin{array}{l}\text { Technical Cha- } \\
\text { racteristics (\#) }\end{array}$ \\
\hline 1. & & & & & $\begin{array}{l}\text { e.g., chemical, } \\
\text { mineralogical } \\
\text { composition, } \\
\text { Los Angeles, } \\
\text { compressive } \\
\text { strength, etc. }\end{array}$ \\
\hline
\end{tabular}

(\#) per recommended application

\section{Secondary Aggregate Production for C\&DW (R2\&R3)}

\begin{tabular}{|l|l|l|l|l|l|}
\hline $\begin{array}{l}\text { Secondary } \\
\text { Aggregate } \\
\begin{array}{l}\text { Processing } \\
\text { Facilities }\end{array}\end{array}$ & $\begin{array}{l}\text { Facility Location } \\
\text { (Coordinates) }\end{array}$ & $\begin{array}{l}\text { Year } \\
\text { Start of } \\
\text { Permit }\end{array}$ & $\begin{array}{l}\text { Year End } \\
\text { of Permit }\end{array}$ & $\begin{array}{l}\text { Type of } \\
\text { Product(s) }\end{array}$ & $\begin{array}{l}\text { Technical Cha- } \\
\text { racteristics (\#) }\end{array}$ \\
\hline 1. & & & & & $\begin{array}{l}\text { e.g., chemical, } \\
\text { mineralogical } \\
\text { composition, } \\
\text { Los Angeles, } \\
\text { compressive } \\
\text { strength, etc. }\end{array}$ \\
\hline
\end{tabular}

(\#) per recommended application

\section{Active Facilities for Secondary Aggregate Production}

\begin{tabular}{|l|l|l|l|l|l|}
\hline Facility $\left(^{*}\right)$ & $\begin{array}{l}\text { List of Active } \\
\text { Facility }\end{array}$ & $\begin{array}{l}\text { Year of re- } \\
\text { porting }\end{array}$ & $\begin{array}{l}\text { Annual Pro- } \\
\text { duction } \\
\text { (tonnes) }(* *)\end{array}$ & $\begin{array}{l}\text { Current } \\
\text { Capacity } \\
\text { (tonnes) }\end{array}$ & \\
\hline 1 & 1 & & & & \\
\hline
\end{tabular}




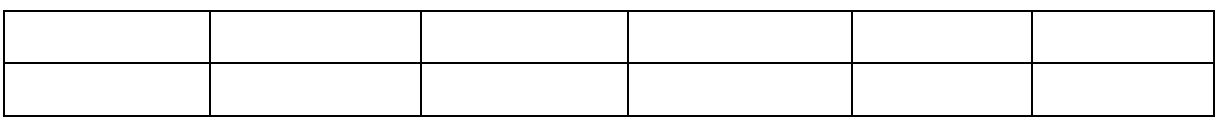

$\left({ }^{*}\right)$ Depends on the Level of Planning, e.g. District, Quarrying area, Region, Municipality, Quarry, etc. 


\section{Analysemethoden}

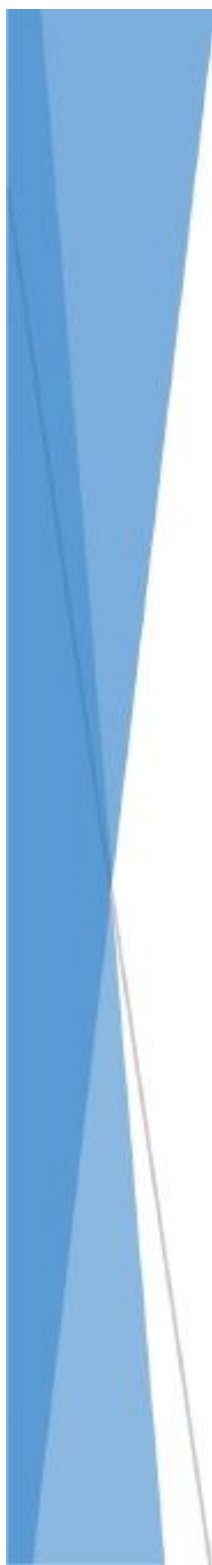

Aktuelle Informationen ist besser als Schätzwerte; zuverlässige Informationen ist die Basis für ein erfolgreiches Gesamtplanungskonzept. Erhalten tatsächlichen und zuverlässige Informa-tion ist eine große Anstrengung. Zur gleichen Zeit ist die Datenüberprüfung ein notwendiger Schritt, um die Zuverlässigkeit und Genauigkeit der gesammelten Informationen zu gewährleisten. Nachfrageprognosen, die den Grundstein für alle Planungsaktivitäten ist, sollten sich auf genaue und zuverlässige Daten beruhen. Bedarfsprognosen können durch eine Vielzahl von Methoden durchgeführt werden, abhängig von den verfügbaren Daten und regelmäßig überarbeitet werden, um sich verändernde wirtschaftliche Bedingungen zu reflektieren. 


\subsection{Einleitung}

Datenerhebung und Speicherung sind große Anstrengungen, die unternommen werden, da die Informationen benötigt wird, um Baurohstoffe Management und Politikplanung zu unterstützen. Qualitätskontrolle, Interpretation, und Vorhersage: Die Analyse der Daten kann in drei große Kategorien unterteilt werden. Qualitätskontrolle ist notwendig, weil Fehler und Versäumnisse in Datenerfassungsprozesse, zB durch Programmierfehler oder Falschmeldungen auftreten können. Ein Ansatz ist, die Stoffströme in der gesamten Baurohstoffe Branche zu analysieren. Daten können in einfachen oder komplexen Modellen analysiert, werden. Eine der wichtigsten Kategorien der Vorhersagemodelle in der Planung verwendet wird, ist der Bedarfsprognose, die wesentliche Vorleistungen für die Entscheidungsfindung liefert.

\subsection{Datenvalidierung}

Wie zuvor erläutert ist, um die Lücke zwischen der Produktion und der effektiven Nachfrage, dh die Menge die voraussichtlich verwendet werden, zu reduzieren; ist es wichtig, dass die Informationen, die für die Planung verwendet Daten sollten so zuverlässig wie möglich sein sollten. Zu diesem Zweck sollte eine Datenvalidierungs Analyse erfolgen; um zu bestätigen, ob Qualitätsanforderungen von Daten (für die Verwendung, die erwartet wird) erfüllen. Nachfolgend werden einige Ansatzpunkte über die Datenprüfung veranschaulicht, insbesondere in Bezug auf die Regelung in Abbildung 3.2. Im Wesentlichen kann der Validierungsprozess horizontal als auch vertikal erfolgen, je nach dem Ziel des Validierungsprozesses, zudem auch in Abhängigkeit von der endgültigen Verwendung der Informationen.

Die horizontale Validierung besteht aus einer Prüfung der Input-Daten aus Fragebögen, Pläne, Projekte oder Formen, aus denen statistische Daten gesammelt und organisiert. Seine kontinuierliche Aktualisierung und Verbesserung stellt ein wesentliches Element, um die Zuverlässigkeit der Daten und damit die Konsistenz der Ergebnisse der Datenanalyse zu verbessern. Durchführung einer horizontalen Validierung eine Überprüfung der Daten auf Plausibilität und, falls notwendig, die Korrektur von Fehlern möglich. Außerdem ist es ein wichtiger Schritt im Lebenszyklus von statistischen Daten, weil es die Zuverlässigkeit der endgültigen Ergebnis der Analyse beeinflusst. In dieser Perspektive kann es nützlich sein, einige Data Quality Goals (DQGs) zu etablieren, was bedeutet, die wünschenswerten Eigenschaften der für die Studie oder die spezifische Branche, für die Daten erhoben werden (zB Zeitabdeckung, geografische Abdeckung, Präzision und Vollständigkeit benötigten Daten , die Quelle der 
Daten, die Unsicherheit von Informationen). Einige spezielle Data Quality Indicators (DQIs) kann für die Festlegung der Qualität der verfügbaren Daten verwendet werden, aber auch Schwellen von gewichteten Werte verwendet werden.

Vertikale Validierung betrifft die Input / Output-Ströme in / aus einem bestimmten Prozesseinheit. Auf diese Weise wird es möglich sein, den Überblick über die Materialien, die in einen Transformationsprozess einbezogen werden zu halten und mögliche Bestände und Verluste. Genauer gesagt, ist diese Art der Analyse auf Massenbilanzen oder der Stoffströme, die von einer Prozesseinheit zum anderen zu bewegen (Abbildung 5.1). Diese Massenbilanzgleichungen berücksichtigen die Menge des Eingangsmaterials in einem bestimmten Prozesseinheit (in der Detail durch die Systemgrenzen Identifikation (Blengini definiert et al., 2014), die Menge des Ausgangsmaterials der gleiche Prozess und die eventuelle Bestände (Materialien, die in das System für einen Zeitraum bleiben und sollte nicht als Abfall oder Verlust berücksichtigt werden).

So beschäftigt sich der erste Schritt bei der Ermittlung der Elementarprozesse und Ströme des Systems unter Analyse. Die Korrelation zwischen Eingabe und Ausgabe stellt den Schritt der Validierung der Daten, da, wenn die Massenbilanz nicht null ist, ist es sofort ersichtlich, ob ein Mangel bezüglich der Verfügbarkeit von Daten und ihrer Genauigkeit.

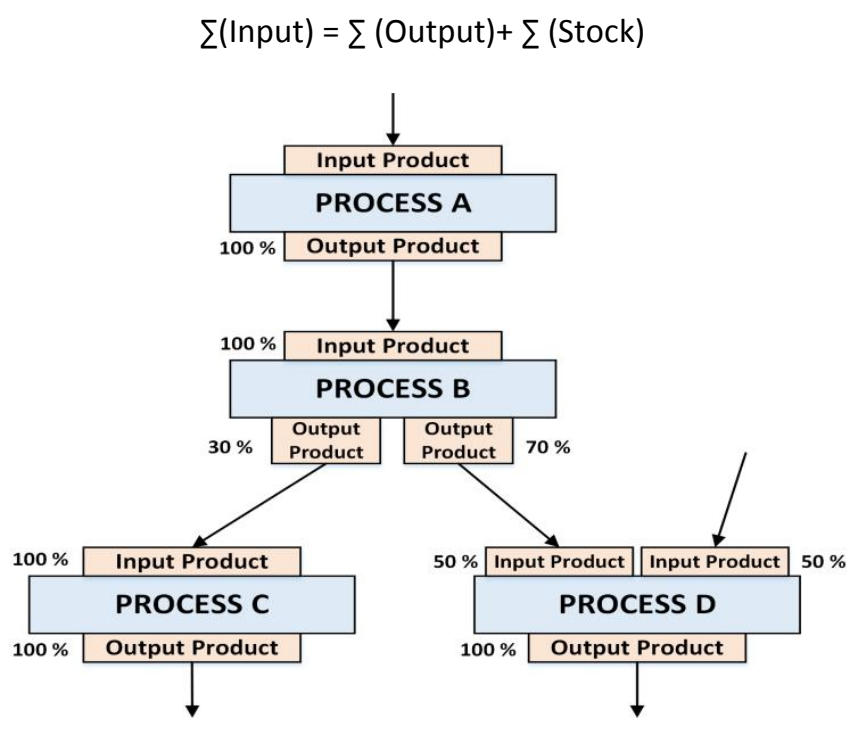

Abb 5.1: Beispiel für die Verbindung zwischen verschiedene Stoffströme 


\subsection{Materialflussrechnung und Analysis}

Materialflussrechnung (MFA) nutzt das Prinzip der im vorangehenden Abschnitt zu studieren eingeführt Massenausgleich, wie Materialien und Energiefluss durch die Wirtschaft und die Umwelt in den Ländern und zwischen den Ländern. Der Zweck der MFA ist Material und Energieflüsse zu verbessern, durch eine Volkswirtschaft und in die Umwelt, als auch für das Verständnis, wie die wirtschaftlichste und ressourceneffiziente Nutzung der Materialien erfolgen kann. MFA verfolgt die Bewegung der Materie in und aus einem System, in diesem Fall Baurohstoffe Sektor. Materialflussanalyse ist die systematische Bewertung der Flüsse von Materialien innerhalb des Systems in einem definierten Raum und Zeit.

\subsection{Nachfrageprognose}

Nachfrage nach Baurohstoffen wird von der Nachfrage nach Endprodukte und Dienstleistungen in der Wirtschaft, wie Wohnen und Verkehr abgeleitet. Treiber der gestiegenen Nachfrage sind Bevölkerungswachstum, zunehmende Lebensstandard, neue oder expandierende Verwendung und Wirtschaftswachstum. Treiber der verringerten Nachfrage nach Baurohstoffe sind höhere Kosten, die zu höheren Preisen führen, höhere Effizienz, wirtschaftliche Bedingungen wie Intensität der Nutzung (in Tonnen pro BIP-Einheit), die typisch ist in entwickelten Volkswirtschaften. Eine weitere mögliche Ursache für verminderte Nachfrage nach Primär Baurohstoffe ist die Substitution, sowie Recycling-Materialien.

Jedes Bedarfsprognose Modell wird seine eigene Komplexität und Detail, Theorie, Methode und Umsetzung haben. Solche, die sektorale Merkmale berücksichtigen, werden als Bottom-up-Modelle bezeichnet; solche auf Basis der Nutzungsintensität von oben nach unten werden ,models' genannt. Sektorale Modelle repräsentieren in der Regel entweder eine aufgestellt Hypothese, Kausalzusammenhang oder basieren auf Zeitreihendaten.

\subsubsection{Kausalnachfrageprognosen auf der Grundlage lokaler Landnutzungsplanung}

Diese Schätzung kann indirekt auf geschätzten Mengen abstellen, unter Berücksichtigung bisheriger, laufender oder geplanter Infrastrukturarbeiten (private Gebäude und öffentliche Arbeiten). Die Instandhaltung, Renovierung und Restaurierung sollte berücksichtigt werden. 


\subsubsection{Kausalbedarfsprognosen auf Basis von Bevölkerungszahl oder Wirtschaftstä- tigkeit}

Der erste dieser Ansätze geht davon aus, daß die Nachfrage für Zuschlagstoffe eine Funktion des Bevölkerungsniveaus ist. Das derzeitige Volumen von Baurohstoffen pro Kopf (m3 / Einwohner) verbraucht als Gesamtproduktion geteilt durch Bevölkerung. Eine Bevölkerungswachstum Projektion wird aus nationalen Statistiken abgeleitet. Wenn Bevölkerung wird gleich bleibt, wird die Nachfrage konstant sein. Wenn Bevölkerung steigt, dann wird die Nachfrage im Verhältnis wachsen. Dieses Verfahren setzt voraus, dass sich Verbrauch weiter erhöht, wie in der Vergangenheit. In der Regel wird diese Methode Markt oder Nachfragetrends nicht berücksichtigen (Cabini \& Zobolu, 2011).

Eine ähnlicher Regressionsansatz basiert auf der Annahme, dass eine Abhängigkeit von der Größe der Wirtschaft basiert; wenn die Wirtschaft wächst, wird die Nachfrage nach Baurohstoffen erhöhen und umgekehrt. Historisch gesehen, war Bruttoinlandsprodukt ein recht guter Indikator für die Nachfrage nach Baurohstoffen, aber dies ist nicht immer der Fall. Erstens ist es nur so lange wirksam, wie Intensität der Nutzung zu nimmt. Allerdings, wenn Nutzungsintensität zu sinken beginnt, führt Wirtschaftswachstum zu weniger statt mehr Konsum. Zweitens wird nicht berücksichtigt, die Verwendung von Ersatzstoffen, wie Recycling-Bau-und Abbruchabfälle (C \& DW).

Es ist auch möglich, komplexere Modelle der Beziehung zwischen Konjunktur und Nachfrage zu konstruieren. Ein ökonometrisches Modell der Wirtschaft könnte auf der Grundlage einer Reihe von linearen oder nichtlinearen Gleichungen erfolgen, die gleichzeitig gelöst werden, wie dies in allgemeinen Gleichgewichtsmodellen getan wird. Oder eine Nachfrageprognose kann durch die Verwendung von System Dynamics Modellierung Simu-nungen erzeugt werden. Die entscheidenden Parametersind Bestände und Ströme in der Wirtschaft, sind die wirtschaftlichen Benchmarks des Bruttoinlandsprodukts (BIP) und Bevölkerung.

\subsubsection{Zeitreihen Prognosen basierend auf Produktionsstatistiken}

"Offizielle" statistische Daten über die Produktion von Zuschlagstoffen werden häufig für die Planung verwendet. Mit Hilfe dieser Daten, nimmt man an, dass die lokale Produktion direkt auf die lokalen Bedürfnisse bezogen wird. Dies kann zu fehlerhaften Vorhersagen für mehrere Gründe führen:

- In Bereichen, in denen die Import und Export Anteile von Baurohstoffen signifikant sind, reflektieren historischen Produktionsmengen nicht die lokale Nachfrage; 
- Produktionsstatistik , die nicht über ein hohes Maß an Zuverlässigkeit beruhen;

- In diesem Fall kann die Art des Materials benötigt nicht geschätzt werden, und somit eine SARM Regelung kann nicht entwickelt werden.

Unter der Annahme, dass die Produktionsstatistiken verfügbar sind, kann ein allgemeiner Trend für die Produktion geschätzt werden, nachdem die jährlichen Daten gefiltert und Fehler beseitigt oder verringert werden. Diese Analyse kann einen Hinweis auf die durchschnittliche Produktion für die kommenden Jahre geben. Es ist sehr wichtig, dass die Trend-Daten durch kontinuierliche Überwachung von Aktivitäten und Vergleiche mit den Ergebnissen der bisherigen Prognosen regelmäßig aktualisiert werden (Cabini \& Zoboli 2011).

\subsubsection{Zeitreihen Prognosen basierend auf Verbrauchsstatistik}

Der Prognoseansatz ist hier methodisch ähnlich wie im vorherigen Abschnitt. Es basiert auf tatsächlichen Gesamtverbrauchswerte für eine bestimmte Region im Laufe der Zeit und auf dem Kontinuitätsprinzip, dh auf der Basis, dass künftiger Verbrauch als einer Extrapolation der aktuellen und früheren Verbrauchsdaten geschätzt werden kann.

Mit historischen Verbrauchsdaten (in der Regel 5 oder 10 Jahre) für diese Analyse, kann es möglich sein, die mittelfristigen Trends, (nach Anpassungen) abzuleiten und somit eine Bedarfsprognose liefern. Die Schwierigkeit in diesem Fall stützt sich in der Verfügbarkeit von konsistenten, verlässlichen und statistisch fundierten Informationen. Wenn einige Daten nicht verfügbar sind, können Schätzungen indirekt durch die Verwendung von Verbrauchsdaten aus anderen Materialien, die zusammen mit Zuschlagstoffen (vor allem Zement) verwendet werden, erfolgen. 


\section{Empfehlungen}

Allgemeine Empfehlungen sind in diesem Abschnitt enthalten.

Länder und Regionen sollten diese Empfehlungen anzupassen, um besser ihren Planungsprozess durchzuführen. 


\subsection{Generelle Empfehlungen}

Allgemeine Empfehlungen in Bezug auf Daten und Datenanalyse für eine nachhaltige Baurohstoff-Planung sind:

- Die Nachfrage nach Baurohstoffen in der SEE-Region sollte von primären und sekundären Baurohstoffe (zB Recycling-Bau-und Abbruchabfälle, Gesteinskörnungen, Aushub von Bauarbeiten, etc.) erfolgen, gem. Ressourceneffizienz Prinzpien.

- $\quad$ Planungsbehörden sollten Mineralressourcen identifizieren in Bezug auf Qualität, Zugänglichkeit und technischen Anforderungen für die Gesamtressourcen in ihrem Bereich, im Rahmen der Erstellung der Entwicklungspläne.

- $\quad$ Es ist wichtig für SEE Ländern / Regionen eine Raumordnungspolitik für Zuschlagstoffe zu etablieren, um Investitionen in den mineralgewinnenden Industrien zu fördern.

- Eine nachhaltige Rohstoffpolitik sollten Rohstoffvorkommen sichern und Raumnutzungsplanung sollte zukünftige Betriebe in diesen Bereichen ermöglichen. Landnutzungskarten sollten auf nationaler, regionaler und lokaler Ebene zur Verfügung stehen.

- $\quad$ Alle betroffenen Interessengruppen, zB solche, die für die Raumplanung und für die Regelung des Planungsprozesses von primären/sekundärenRohstoffen; Industrie, Gemeinden, NGOs und der Zivilgesellschaft, sollten in enger Zusammenarbeit kooperieren.

- Baurohstoff-Planung sollte in vorgegebenen Zeitabständen überprüft werden.

- $\quad$ SEE-Ländern / Regionen sollten den Austausch von Daten für Baurohstoffplanung sondieren, in Bezug auf Import- und Exportoptionen zwischen Nachbarländern.

\subsection{Empfehlungen für Daten und Datenanalysemethoden}

Spezifische Empfehlungen für die Datensammlung, Validierung und Analyse zu verbessern sind: 
Effiziente Verfahren der Datenerhebung,-Management und Datenverarbeitung sollte für die Entwicklung der SARM (Sustainable Aggregates Resource Management) und SSM Konzepte (Sustainable Supply Mix) festgelegt werden.

Die Entscheidungsfindung sollte auf den besten verfügbaren, wissenschaftlich fundierten Daten basieren. Daten sollten gründlich überprüft und vor der Verarbeitung validatiert werden.

$>$ Regelmäßige Untersuchungen sollten durchgeführt werden, die detaillierte Informationen bieten bez. Reserven und Ressourcen, Konsum und Produktionsvolumen, Regionalströme, Transport, bewilligte Reserven in Abhängigkeit der Planungsebene.

$>$ Ein Informationsmatrix sollte für jede Planungs-Ebene identifizieren werden in Bezug auf Prozesse und Datensätzen.

> Bedarfsprognosen sollten mit der am besten geeigneten Methode in jeder Region / Land erfolgen. Informationen hinsichtlich öffentliche Arbeiten, privaten Sektor, Exporte, usw. sollten bereitgestellt werden.

A Alle Daten sollten in einer elektronischen Datenbank organisiert werden, die regelmäßig aktualisiert wird. Als Beispiel soll ein Verzeichnis der aktiven und inaktiven (erlaubt) Abbaue, inkl. Produktionskapazität, in der Datenbank geführt werden.

Die Daten sollten, um die Zuverlässigkeit der Informationen zu gewährleisten validiert werden. 


\section{Referenzen}

Agioutantis Z., Komnitsas, K.\& Athousaki, A. (2013). Aggregate Transport and Utilization in Urban Areas: Ecological Footprint and Environmental Impacts, Proceedings of the 13th International Congress, Chania, Greece, 5-8 Sept 2013, Bulletin of the Geological Society of Greece, vol. XLVII 2013.

Austria, (2014). http://www.en.bmwfw.gv.at/Energy/Seiten/TheAustrianMineralResourcesPlan.aspx

BGS (2014a). http://www.bgs.ac.uk/planning4minerals/Glossary.htm

BGS (2014b). http://www.bgs.ac.uk/planning4minerals/Resources_4.htm

BGS (2014c). http://www.bgs.ac.uk/planning4minerals/Resources_5.htm

BGS, (2012). "Aggregates safeguarding maps of Wales", Keyworth, Nottingham, British Geological Survey.

Blengini, G.A., Moltrer, A., Valbusa, M., Komnitsas, K., Agioutantis, Z. \& Garbarino, E. (2013). Data and methodologies for a resource-efficient planning of primary and secondary aggregates in South East Europe (SEE) countries, Proceedings, 6th International Conference on Sustainable Development Indicators in the Minerals Industry (SDIMI 2013), 30 June - 3 July 2013, Milos, Greece, pp. 282-288.

Blengini G.A., Bobba S., Shields D.J., Moltrer A. \& Valbusa M. (2014). Report on Data Analysis Methodologies, SNAP-SEE project.

Cabini, E., \& Zoboli, R. (2011). Ricerca per il dimensionamento dei volumi di sostanze minerali di cava per il nuovo Piano cave 2013-2023 della Provincia di Cremona. Alta Scuola per l'Ambiente - Università Cattolica di Brescia.

Chalkiopoulou, F. \& Hatzilazaridou, K. (2011). How to achieve aggregates resource efficiency in local communities, a joint manual, for stakeholders' decision making on the local level, SARMa Manual, http://www.sarmaproject.eu.

Hatzilazaridou, K. (2014). Data Dictionary, SNAP-SEE, http://www.snapsee.eu.

Leoben (2010). Planning Policies and Permitting Procedures to Ensure the Sustainable Supply of Aggregates in Europe, Final Report, Commissioned by UEPG, Department of Mineral Resources and Petroleum Engineering, University of Leoben, Austria, June.

Mazzanti, M., Paleari, S.\& Zoboli, R. (2007). Effectiveness of environmental taxes and charges for managing sand, gravel and rock extraction in selected EU countries Italy. 
SA, (2014). http://www.sustainableaggregates.com/sourcesof aggregates/recycle/ SARMa (2011). http://www.sarmaproject.eu/

SEA, (2001). Directive 2001/42/EC "on the assessment of effects of certain plans and programmes on the environment"

SPP, (2006). Scottish Planning Policy SPP4 "Planning for Minerals", Scottish Executive, www.scotland.gov.uk/publications/2006/08/30152427].

Springer (2014).

http://www.springerreference.com/docs/html/chapterdbid/349568.html.

UEPG Annual

Review

2012-2013.

http://www.uepg.eu/uploads/Modules/Publications/uepg-ar2012-

2013_en_inter_v14_pbp_small.pdf

UK

Gov

(2014).

https://www.gov.uk/government/uploads/system/uploads/attachment_data/file/6286/forecastingaggregatesdemand.pdf

Welsh Assembly Government, Department for Communities and Local Government (2011) "Collation of the results of the 2009 aggregate minerals survey for England and Wales", Second Edition, October 2011.

Worchestershire County Council, (2013). Minerals Local Plan Background Document. Worcestershire Local Aggregates Assessment, http://www.worcestershire.gov.uk / mineralsbackground

http://www.aggregate.com/glossary-of-terms/\#sthash.oqjYPj46.dpuf

http://www.businessdictionary.com/definition/demand-forecast.html

http://economics.about.com/cs/economicsglossary/g/demand.htm

http://www.oecd.org/environment/indicators-modelling-outlooks/MFA-Guide.pdf

http://www.scotland.gov.uk/publications/2010/02/03132605/12

http://www.scotland.gov.uk/publications/2006/08/30152427/0 


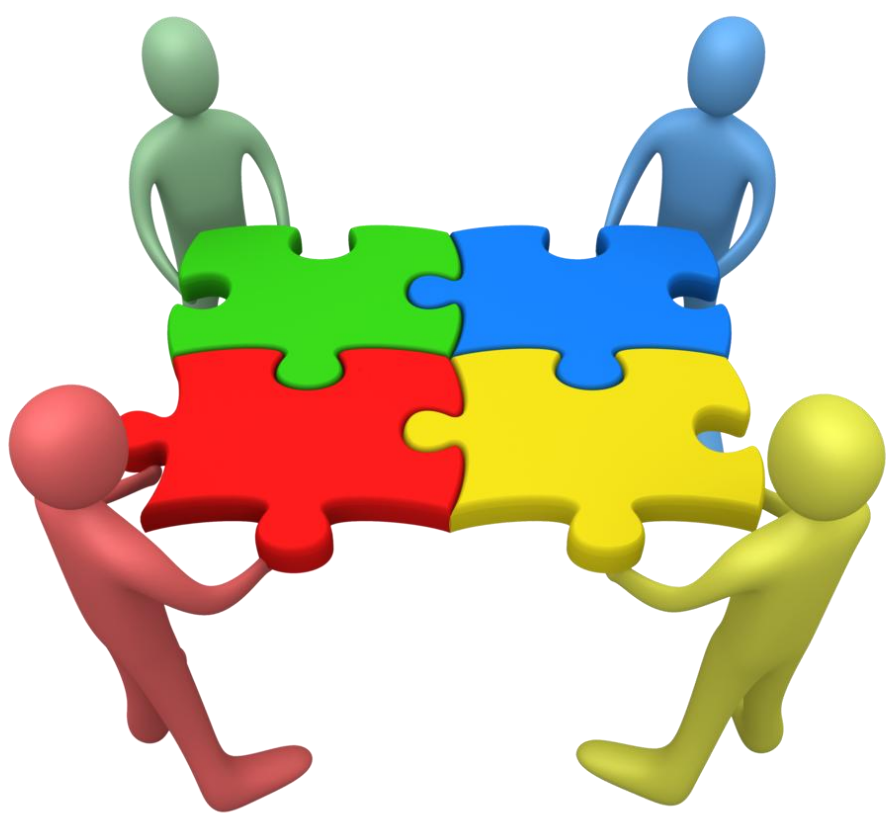

Sustainable Aggregates Planning in South East Europe (SNAP-SEE) 